\title{
A CHARACTERIZATION OF CELLULAR ARCS IN EUCLIDEAN 3-SPACE
}

\author{
FRANCIS D. LONERGAN
}

1. Introduction. As in [8], a topological space $X$ is said to have a contractible basis at a point $x$ in $X$ if and only if there exists a basis $G_{x}=\left\{U_{x}\right\}$ of open neighborhoods of $x$ with the property that each $U_{x}$ is contractible (relative to some point) and furthermore $U_{x}-x$ is both pathwise and simply connected. The main result in this note is the following: An arc $\alpha$ in euclidean 3-space, $E^{3}$, is cellular if and only if the quotient space $X=E^{3} \bmod \alpha$ obtained by collapsing $\alpha$ to a point has a contractible basis at the image $p(\alpha)=x$ (point) of $\alpha$ under the natural projection $p$ of $E^{3}$ on to $X$.

Examples 1.1., 1.1* and 1.3 in [1], afford examples of noncellular $\operatorname{arcs}$ in $E^{3}$.

2. Main result. The definition of a combinatorial $n$-manifold can be found in [9, p. 290].

Lemma 1. If $\alpha$ is an arc in $E^{3}$, then $\alpha$ has arbitrarily small open neighborhoods with connected boundaries.

PRoof. Let $\epsilon>0$ be assigned and $U_{\epsilon}(\alpha)$ the open $\epsilon$-neighborhood of $\alpha$. Take a triangulation of $E^{3}$ of mesh $<\epsilon / 3$ and let $N\left(\alpha, E^{3}\right)$ be the (connected) simplicial neighborhood of $\alpha$ relative to this triangulation. Take a regular neighborhood (connected), $M^{3}$, of $N\left(\alpha, E^{3}\right)$ lying within the open $\epsilon / 3$-neighborhood of $N\left(\alpha, E^{3}\right)$. Then $M^{3}$ is a compact, connected, combinatorial 3-manifold with boundary $B$ and $\alpha \subseteq$ interior of $M^{3}=M^{3}-B$. Suppose $B_{1}, \cdots, B_{n}, n \geqq 2$, are the components of $B$. Under a suitable successive barycenter subdivision of $M^{3}$, there exist vertices $a_{2}, \cdots, a_{n}$ in $B_{1}$, vertices $b_{2}, \cdots, b_{n}$ in $B_{2}, \cdots, B_{n}$ respectively, and a pairwise disjoint collection of simplicial arcs $\beta_{2}, \cdots, \beta_{n}$ (which do not meet $\alpha$ ) from $a_{2}$ to $b_{2}, \cdots, a_{n}$ to $b_{n}$ respectively such that for each $j, j=2, \cdots, n, \beta_{j}-\left(a_{j} \cup b_{j}\right)$ $\subseteq M^{3}-B$. Since for $j=2, \cdots, n, \beta_{j}, a_{j}$ and $b_{j}$ are geometrically collapsible, there exists (under a suitable subdivision of $M^{3}$ ) a pairwise disjoint collection of simplicial neighborhoods $N_{2}, \cdots, N_{n}$ of $\beta_{2}$, $\cdots, \beta_{n}$ respectively which are combinatorial 3-cells (3-elements) with the property that for $j=2, \cdots, n, N_{j} \cap B_{j}=D_{j}$ and $N_{j} \cap B_{1}$ $=D_{j 1}$ are combinatorial 2-cells, $N_{j} \cap B_{i}=\varnothing$ (empty set) for $i \neq 1$, $j \neq i$ and $\alpha \subseteq \stackrel{\circ}{M}_{1}^{3}=M^{3}-\left(B \cup \cup_{j=2}^{n} N_{j}\right)$ which is open in $E^{3}$. By a point-

Received by the editors September 11, 1966. 
set argument one can show that the boundary, $B^{1}$, of $\stackrel{\circ}{M}_{1}^{3}$ is connected where, $B^{1}=\left[B_{1}-\bigcup_{j=2}^{n}\right.$ int $\left.D_{j 1}\right] \cup\left[\bigcup_{j=2}^{n}\left(B_{j}-\right.\right.$ int $\left.\left.D_{j}\right)\right] \cup\left[\bigcup_{j=2}^{n}\left(N_{j}-\right.\right.$ int $\left.\left.N_{j}\right)\right]$, with int $D_{j 1}=$ interior of $D_{j 1}$ relative to $B_{1}$; int $D_{j}=$ interior of $D_{j}$ relative to $B_{j}$; int $N_{j}=$ interior of $N_{j}$ relative to $M^{3}$. Thus, $\alpha \subseteq \stackrel{\circ}{M}_{1}^{3}$ $\subseteq \stackrel{\circ}{M}_{1}^{3} \cup B^{1} \subseteq M^{3} \subseteq U_{\mathrm{\epsilon}}(\alpha)$.

TheOREM 1. An arc $\alpha$ in $E^{3}$ is cellular if and only if $X=E^{3} \bmod \alpha$ has a contractible basis at $p(\alpha)=x$ (point).

Proof. If $\alpha$ is cellular, then $X$ is homeomorphic to $E^{3}$ and so is locally euclidean [2]. Conversely, if $X$ has a contractible basis at $x$ then since $x$ has arbitrarily small open neighborhoods with connected boundaries (since $\alpha$ has this property by Lemma 1) and $X$ is a singular homology manifold with integers as coefficients [6], [7], it follows by the proof of Theorem II.3 in [8], that $X$ is a (strong) homotopy manifold as defined in [3]. Hence by the proof of the Main Theorem in $[3], \alpha$ is a cellular arc.

\section{Bibliography}

1. E. Artin and R. H. Fox, Some wild cells and spheres in 3-dimensional Euclidean space, Ann. of Math. 49 (1948), 979-990.

2. N. Brown, $A$ proof of the generalized Schoenflies theorem, Bull. Amer. Math. Soc. 66 (1960), 74-76.

3. M. L. Curtis, Shrinking continua in 3-space, Proc. Cambridge Philos. Soc. 57 (1961), 423-433.

4. P. J. Hilton and S. Wylie, Homology theory, Cambridge Univ. Press, Cambridge, 1960.

5. W. Hurewicz and H. Wallman, Dimension theory, Princeton Univ. Press, Princeton, N. J., 1948.

6. F. Raymond, Poincare duality for homology manifolds, Doctoral dissertation, Univ. of Michigan, Ann Arbor, 1958.

7. - Some remarks on the coefficients used in the theory of homology manifolds, Pacific J. Math. 15 (1965), 1365-1376.

8. P. M. Rice, Homotopically homogeneous spaces and manifolds, Trans. Amer. Math Soc. 120 (1965), 247-254.

9. J. H. C. Whitehead, Simplicial spaces, nuclei, and m-groups, Proc. London Math. Soc. 45 (1939), 243-327.

Florida State University 